\title{
Uber die Beschränktheit der Lösungen diskreter nichtstationärer Phasensysteme
}

\section{RETTMANN}

Es werden hinreichende Bedingungen für die Beschränktheit der Lösungen und für $\Delta$-Stabilität im ganzen von diskreten Phasensystemen formuliert, die enteprechende Ergebnisse von $Y_{U}$. A. Koryakn, G. A. Leonov, A. R. LISS, V. N. BeLYkH, V. P. Maksakov [3, 5-7] verallgemeinern.

Приведены достаточные условия ограниченвости решений и $\Delta$-устоичивости в целом дискретных фазовых систем, которые обобщают соответствующие результаты Ю. А. КорякинА, Г. А. ЛЕоновА, А. Р. ЛисСА, В. Н. БеЛИХ, В. П. МАнСАКовА [3, 5-7].

In this paper we give sufficient conditions for the solutions to be boundet and for the $\Delta$-stability in the whole of discrete phase systems. This generalizis related results of Yס. A. KonYAKIN, G. A. Leonov, A. R. LISS, V. N. BeLYKH, V. P. MAKSAKov [3, 5-7].

In der vorliegenden Arbeit werden diskrete Phasensysteme, d. h. Systemè der automatischen Steuerung mit einer bezüglich bestimmter Argumente periodischen Nichtlinearität, mit Methoden der absoluten Stabilitätstheorie betrachtet und damit in $[1,3,5-8,14]$ angegebene Untersuchungen fortgeführt. Dabei wird zunächst ein Frequenzkriterium für die Beschränktheit der Lösungen diskreter Phasensysteme mit einer Nichtlinearität, die auch unstetig sein kann; bewiesen. Unstetige Nichtlinearitäten spielen z. B. bei der Beschreibung von Ziffernsystemen der Phasensynchronisation eine Rolle.

Einen wichtigen Platz beim Nachweis der Beschränktheit der Lösungen nimmt die Betrachtung spezieller Matrizenungleichungen ein. Ihre Lösbarkeit wird durch den „Frequenzsatz" [16] (mitunter auch als „Lemma von YARUBovioH-KaLmax" bezeichnet) garantiert. Mit Hilfe dieser Matrizenungleichungen wird im Phasenraum der zu untersuchenden Systeme ein Netz von quadratischen Kegeln konstruiert, die invariant für die Lösungen der Systeme sind. Weiterhin wird ein Kriterium für die Beschränktheit der Lösungen von diskreten Phasensystemen formuliert, das keinen „Frequenzcharakter" trägt, sondern auf der Benutzung eines Netzes invarianter Kegel, die als Schnitt einer endlichen Zahl von Hyperebenen entstehen, beruht. Vom praktischen Standpunkt aus garantiert die Beschränktheit der Lösungen von Systemen der Phasensynchronisation einen Synchronismus ,,im Mittel“".

Schließlich wird in der Arbeit ein Kriterium für die $\Delta$-Stabilität im ganzen von diskreten Phasensystemen angegeben.

Mit Hilfe der formulierten Kriterien werden in der Arbeit die Beschränktheit der Lösungen und die $\Delta$-Stabilität im ganzen eines autonomen Ziffernphasenkopplungs-. systems erster Ordnung, in dessen Steuerkette der Ziffernfilter fehlt, nachgewiesen.

Der Charakter der in der Arbeit erhaltenen Ergebnisse und die Vorgehensweise besitzen weitgehende. Ahnlichkeit mit Untersuchungen von kontinuierlichen Phasensystemen, die durch nichtlineare Differentialgleichungssysteme beschrieben werden. Die Spezifik diskreter Systeme kommt jedoch insbesondere in Lemma 2 
zum Ausdruck, in dem eine Art Sprungverhalten diskreter Systeme charakterisiert wird, das bei kontinuierlichen Steuersystemen nicht anzutreffen ist. Deshalb lassen sich z. B. in diskreten Systemen erster Ordnung Schwingungen im Sinne von YaxUBovich nachweisen [12], während bekanntlich [1] in Differentialgleichungen erster Ordnung Schwingungen nicht auftreten können.

Gegeben sei das System

$$
\begin{aligned}
& x_{t+1}=A x_{t}+b \varphi\left(t, \sigma_{t}\right), \\
& \sigma_{t}=c^{*} x_{t} \quad(t=0,1, \ldots) .
\end{aligned}
$$

mit der konstanten $v \times v$-Matrix $A$, die den Eigenwert 1 und $v-1$ Eigenwerte innerhalb des offenen Einheitskreises besitzt. Des weiteren sind $b$ und $c$ konstante $\nu$-Vektorẹ, für die das $\operatorname{Paar}(A, b)$ vollkommen steuerbar ist und das $\operatorname{Paar}(A, c)$ vollkommen beobachtbar ist. $\left.{ }^{1}\right)$ Mit dem Symbol * wird die Operation des Transponierens bezeichnet. Alle in der Arbeit vorkommenden Größen sind, soweit nicht anders vereinbart, reell. Es sei $\varphi(t, \sigma)$ eine skalare Funktion der Argumente $t \in\{0,1, \ldots\}$ und $\sigma \in \mathbf{R}^{1}$, die der Bedingung

$$
\mu_{1} \sigma^{2} \leqq \varphi(t, \sigma) \cdot \sigma \leqq \mu_{2} \sigma^{2} \quad\left(\sigma \in \mathbf{R}^{1}, t=0,1, \ldots\right)
$$

genügt. Dabei sind $\mu_{1}$ und $\mu_{2}$ Konstanten mit

$$
-\infty \leqq \mu_{1} \leqq 0<\mu_{2} \leqq+\infty \text {. }
$$

Es sei außerdem die Funktion $\varphi$ periodisch bezüglich $\sigma$ mit der Periode $\Delta>0$, d. h., es gelte

$$
\varphi(t, \sigma+\Delta)=\varphi(t, \sigma) \quad\left(\sigma \in \mathbf{R}^{1}, t=0,1, \ldots\right) .
$$

Unter den getroffenen Voraussetzungen bezüglich des linearen Teils des Systems (1) existiert ein Vektor $r$ mit . $\quad:$

$$
A r=r \text { und } c^{*} r=\Delta .
$$

Zum Nachweis der Richtigkeit dieser Behauptung ist es ausreichend zu zeigen, daß für den Eigenvektor $r$ von $A$ mit $A r=r$ (ein solcher existiert laut Voraussetzung) $c^{*} r \neq 0$ gilt. Wenn man annimmt, daß $c^{*} r=0$ ist, so erhält man die Beziehungen

$$
c^{*} A^{k} r=c^{*} r=0 \quad(k=0,1, \ldots, v-1),
$$

woraus wegen der vollständigen Beobachtbarkeit des Paars $(A, c)$ die Gleichung $r=0$ folgt. Der erhaltene Widerspruch beweist die Möglichkeit der Darstellung (5).

Wir bezeichnen mit

$$
\chi(p)=c^{*}(A-p I)^{-1} b
$$

die Ubertragungsfunktion des linearen Teils von System (1) vom Eingang $\varphi$ zum Ausgang $-\sigma$. Dabei ist $p$ eine komplexe Zahl und $I$ die $v \times v$-Einheitsmatrix.

Satz 1. Es existiere eine Zahl $\lambda \in(0,1)$, so da $\beta$ die Matrix $\frac{1}{\lambda} A$ einen Eigenwert außerhalb des Einheitskreises und $v-1$ Eigenwerte innerhalb des offenen Einheits:

1) Das Paar $(A, b)$ heiBt vollkommen steuerbar, wenn $\operatorname{det}\left\|b, A b, \ldots, A^{y-1} b\right\| \neq 0$ ist. Das Paar $(A, c)$ heißt vollkommen beobachtbar, wenn $\operatorname{det}\left\|c, A^{*} c, \ldots,\left(A^{*}\right)^{*-1} c\right\|=0$ ist. Dabei bezeichnet *, wie auch oben, die Operation des Transponierens. 
kreises besitzt und für alle komplexen $p$ mit $|p|=1$ gilt: ${ }^{2}$ )

$$
\begin{array}{lll}
\operatorname{Re}\left\{\left[1+\mu_{1} \chi(\lambda p)\right]\left[1+\mu_{2} \chi^{*}(\lambda p)\right]\right\}>0 & \text { bei } \mu_{1} \neq-\infty, & \mu_{2} \neq+\infty, \\
\operatorname{Re}\left\{\chi(\lambda p)\left[1+\mu_{2} \chi^{*}(\lambda p)\right]\right\}<0 & \text { bei } \mu_{1}=-\infty, & \mu_{2} \neq+\infty, \\
\operatorname{Re}\left\{\left[1+\mu_{1} \chi(\lambda p)\right] \chi^{*}(\lambda p)\right\}>0 & \text { bei } \mu_{1} \neq-\infty, & \mu_{2}=+\infty
\end{array}
$$

Dann ist jede Lösung des Systems (1) beschränkt auf $[0,+\infty)$.

Für den Beweis dieses Satzes sind eine Reihe von Hilfsaussagen notwendig, die im weiteren formuliert und bewiesen werden.

Lemma 1. Es sei $Q$ eine $v \times v$-Matrix, $q$ und $r$ seien $v$-Vektoren, wobei $q \neq 0$ ist. Es sei weiterhin

$$
\mathfrak{S}(x, \xi)=x^{*} G_{0} x+2 g r^{*} x \xi
$$

eine quadratische Form der Veränderlichen $x \in \mathbf{R}^{*}$ und $\boldsymbol{\xi} \in \mathbf{R}^{\mathbf{1}}$ : Dabei ist $G_{0}=G_{0}{ }^{*}$ eine positiv semidefinite $\nu \times \nu$-Matrix, $g$ ist eine Zahl. Es existiere eine $\nu \times \nu$-Matrix $H=H^{*}$, die einen negativen und $v-1$ positive Eigenwerte besitzt, und es existiere eine Zahl $\delta>0$, so daß die Ungleichung ${ }^{3}$ )

$$
\begin{aligned}
& (Q x+q \xi)^{*} H(Q x+q \xi)-x^{*} H x+\left(\mathfrak{G}(x, \xi) \leqq-\delta|x|^{2}\right. \\
& \left(x \in \mathbf{R}^{*}, \xi \in \mathbf{R}^{1}\right)
\end{aligned}
$$

erfüllt ist. Dann gilt

$$
\left\{x \mid x^{*} H x \leqq 0\right\} \cap\left\{x \mid r^{*} x=0\right\}=\{0\} .
$$

Beweis. Wegen der Eigenwertverteilung von $H$ existiert ([4], S. 297) ein Vektor $h$, so daß gilt:

$$
\left\{x \mid x^{*} H x \leqq 0\right\} \cap\left\{x \mid h^{*} x=0\right\}=\{0\} .
$$

Wird in der Ungleichung (6) $x=0$ gesetzt, erhält man $q^{*} H q \leqq 0$. Hieraus und aus (8) folgt $h^{*} q \neq 0$. Es werde angenommen, daß (7) nicht erfüllt ist, d. $h .$, daß es einen Vektor $x_{0} \neq 0$ mit $r^{*} x_{0}=0$ und $x_{0}^{*} H x_{0} \leqq 0$ gibt. Wegen $x_{0}^{*} G_{0} x_{0} \geqq 0$ erhalten wir dann aus (6) die Ungleichung

$$
\left(Q x_{0}+q \xi\right)^{*} H\left(Q x_{0}+q \xi\right) \leqq-\delta\left|x_{0}\right|^{2}<0 \quad\left(\xi \in \mathbf{R}^{1}\right) .
$$

Wegen $h^{*} q \neq 0$ läßt sich ein solches $\xi_{0}$ angeben, daß

$$
h^{*}\left[Q x_{0}+q \xi_{0}\right]=0
$$

gilt. Aus (8), (9) und (10) folgt sofort

$$
Q x_{0}+q \xi_{0}=0 \text {. }
$$

Es ist offensichtlich, $\mathrm{da} B$ die Beziehungen (9) und (11) einander widersprechen. Lemma 1 ist bewiesen.

Bemerkung. Lemma 1 wurde unter etwas anderen Voraussetzungen bereits in [13] bewiesen. In [7] wurde dieses Lemma zur Herleitung von Frequenzkriterien der Stabilität diskreter Systeme, die die Dynamik von Ziffernsystemen der Phasensynchronisation beschreiben, benutzt.

2) Im Satz 1 dient das Symbol * ausnahmsweise auch zur Kennzeichnung der konjugiert komplexen Zahl.

s) Hier und im weiteren ist $|x|$ die Euklidische Norm des Vektors $x \in \mathbf{R}$. 
Lemma 2. Wir betrachten das System

$$
\begin{aligned}
& x_{t+1}=Q x_{t}+q \varphi\left(t, \sigma_{t}\right), \\
& \sigma_{t}=r^{*} x_{t} \quad(t=0,1, \ldots) .
\end{aligned}
$$

Hierbei ist $Q$ eine $\nu \times \nu$-Matrix; $q$ und $r$ sind $\nu$-Vektoren. Das Paar $(Q, r)$ sei vollkommen beobachtbar und $\varphi$ sei eine skalare Funktion der Argumente $t \in\{0,1, \ldots\}$ und $\sigma \in \mathbf{R}^{\mathbf{1}}$, die den Beziehungen (2) und (3) genügt. Es mögen weiterhin eine Matrix $H=H^{*}$ und Zahlen $\lambda>0$ und $\delta>0$ existieren, für die gilt:

$$
\frac{1}{\lambda^{2}}(Q x+q \xi)^{*} H(Q x+q \xi)-x^{*} H x+\left(S(\sigma, \xi) \leqq-\delta|x|^{2}\left(x \in \mathbf{R}^{\prime}, \xi \in \mathbf{R}^{1}\right) .\right.
$$

Dabei ist $\sigma=r^{*} x$ und

$$
\mathcal{S}(\sigma, \xi)= \begin{cases}\left(\mu_{2} \sigma-\xi\right)\left(\xi-\mu_{1} \sigma\right) & \text { für } \mu_{1} \neq-\infty, \mu_{2} \neq+\infty, \\ \left(\mu_{2} \sigma-\xi\right) \sigma & \text { für } \mu_{1}=-\infty, \mu_{2} \neq+\infty, \\ \left(\xi-\mu_{1} \sigma\right) \sigma & \text { für } \mu_{1} \neq-\infty, \mu_{2}=+\infty .\end{cases}
$$

Es sei h ein $\nu$-Vektor, für den (zusammen mit $H$ aus (13)) die Beziehung (8) gilt. Wir bezeichnen: $\Gamma=\left\{x \mid x^{*} H x<0\right\}, \Gamma^{+}=\Gamma \cap\left\{x \mid h^{*} x \geqq 0\right\}$ und $\Gamma^{-}=\Gamma \cap\left\{x \mid h^{*} x \leqq 0\right\}$. Sei $x_{t}\left(t_{0}, x_{0}\right)$ eine Lösung des Systems (12) mit der Anfangsbedingung $x_{t_{0}}\left(t_{0}, x_{0}\right)=x_{0}$. Dann gilt:

1. Wenn die Matrix $\frac{1}{\lambda} Q$ einen (reellen) Eigenwert $>1$ und $v-1$ Eigenwerte innerhalb des offenen Einheitskreises hat, so ist für die Lösungen von System (12) erfüllt:

a) aus $x_{0} \in \Gamma^{+}$folgt $x_{t}\left(t_{0}, x_{0}\right) \in \Gamma^{+}\left(t \geqq t_{0}\right)$;

b) aus $x_{0} \in \Gamma^{-}$folgt $x_{t}\left(t_{0}, x_{0}\right) \in \Gamma^{-}\left(t \geqq t_{0}\right)$.

2. Wenn die Matrix $\frac{1}{\lambda} Q$ einen (reellen) Eigenwert $<-1$ und $v-1$ Eigenwerte innerhalb des offenen Einheitskreises hat, so ist für die Lösungen von System (12) erfüllt:

a) aus $x_{0} \in \Gamma^{+}$folgt

$$
x_{\ell_{0+2 k}}\left(t_{0}, x_{0}\right) \in \Gamma^{+} \text {und } x_{\ell_{0}+2 k+1}\left(t_{0}, x_{0}\right) \in \Gamma^{-} \quad(k=0,1, \ldots) \text {; }
$$

b) aus $x_{0} \in \Gamma^{-}$folgt

$$
x_{t_{0}+2 k}\left(t_{0}, x_{0}\right) \in \Gamma^{-} \text {und } x_{t_{0}+2 k+1}\left(t_{0}, x_{0}\right) \in \Gamma^{+} \quad(k=0,1, \ldots) .
$$

Beweis. Wird in (13) $\xi=0$ gesetzt und beachtet, daß wegen (2) und (3) $\left(\mathfrak{S}\left(r^{*} x, 0\right) \geqq 0\left(x \in \mathbf{R}^{0}\right)\right.$ gilt, so erhält man aus (13) die Beziehung

$$
\frac{1}{\lambda^{2}} x^{*} Q^{*} H Q x-x^{*} H x \leqq-\delta|x|^{2} \quad\left(x \in \mathbf{R}^{\prime}\right) \text {. }
$$

Es sei $\frac{x}{\lambda}$ der Eigenwert von $\frac{1}{\lambda} Q$, der außerhalb des Einheitskreises liegt. Wird in der letzten Ungleichung $x=u$ gesetzt, wobei $u$ ein Eigenvektor ist, der zum Eigenwert $\varkappa$ der Matrix $Q$ gehört, so erhält man

$$
\left(\frac{x^{2}}{\lambda^{2}}-1\right) u^{*} H u \leqq-\delta|u|^{2}
$$

Hieraus folgt

$$
u^{*} H u<0 \text {. }
$$


Aus (16) und (8) folgt $h^{*} u \neq 0$. Deshalb kann o. B. d. A. angenommen werden, daß

$$
h^{*} u>0
$$

gilt. Weiterhin ist wegen der Beobachtbarkeit von $(Q, r)$

$$
r^{*} u \neq 0 \text {. }
$$

(Der Nachweis érfolgt wie bei. (5).) Wir beweisen nun die Beziehung (15) indirekt. Es werde angenommen, da $\beta$ ein Punkt $x_{0} \in \Gamma^{+}$und eine Zahl $t_{0} \geqq 0$ existieren, für die gilt:

$$
Q x_{0}+q \varphi\left(t_{0}, r^{*} x_{0}\right) \notin \Gamma^{+} .
$$

Aus (2) und (3) folgt, $\operatorname{daB} \mathfrak{S S}\left(r^{*} x_{0}, \varphi\left(t_{0}, r^{*} x_{0}\right)\right) \geqq 0$ ist und deshalb wegen (13) $Q x_{0}+q \varphi\left(t_{0}, r^{*} x_{0}\right) \in \Gamma$ gilt. Folglich ist (19) nur möglich, wenn

$$
h^{*}\left[Q x_{0}+q \varphi\left(t_{0}, r^{*} x_{0}\right)\right]<0
$$

ist. Wegen (18) kann eine solche $Z a h l ~ \varrho>0$ gewählt werden, daß für den Punkt $x_{1}=\varrho u$ gilt : $r^{*} x_{1} \neq r^{*} x_{0}$. Es wird nun eine für alle $\sigma \in \mathbf{R}^{1}$ definierte stetige Hilfsfunktion $\tilde{\varphi}(\sigma)$ eingeführt, für die gelte:

$$
\tilde{\varphi}\left(r^{*} x_{1}\right)=0, \quad \tilde{\varphi}\left(r^{*} x_{0}\right)=\varphi\left(t_{0}, r^{*} x_{0}\right) \quad \text { und } \quad \mathcal{H}(\sigma, \tilde{\varphi}(\sigma)) \geqq 0 \quad\left(\sigma \in \mathbf{R}^{1}\right) .
$$

Eine solche Festlegung ist möglich, da $\{(\sigma, \xi) \mid \mathfrak{S}(\sigma, \xi) \geqq 0\}$ eine zusammenhängende Menge ist und für alle $\sigma \in \mathbf{R}^{1}$ die Punkte $(\sigma, 0)$ wegen (3) und (14) dieser Menge angehören. Die Menge $\Gamma^{+}$ist wegen (8) und [13] (Hilfssatz 1) konvex. Folglich gilt

$$
y(s)=s x_{0}+(1-s) x_{1} \in \Gamma^{+} \quad(s \in[0,1]) .
$$

Wegen $h^{*} y(s)=s h^{*} x_{0}+(1-s) h^{*} x_{1}>0(s \in[0,1]),(8)$ und (21) ist

$$
y(s) \neq 0 \quad(s \in[0,1]) \text {. }
$$

Aus (13) folgt wegen $(S)\left(r^{*} y(s), \tilde{\varphi}\left(r^{*} y(s)\right)\right) \geqq 0(s \in[0,1])$ und (21) die Ungleichung

$$
\left[Q y(s)+q \tilde{\varphi}\left(r^{*} y(s)\right)\right]^{*} H\left[Q y(s)+q \tilde{q}\left(r^{*} y(s)\right)\right] \leqq-\delta|y(s)|^{2} \quad(s \in[0,1]) \text {. }
$$

Aus (22) und (23) ergibt sich

$$
Q y(s)+q \tilde{\varphi}\left(r^{*} y(s)\right) \neq 0 \quad(s \in[0,1]) . \quad .
$$

Wir benutzen nun die stetige Funktion

$$
f(s)=h^{*}\left[Q y(s)+q \tilde{\varphi}\left(r^{*} y(s)\right)\right] \quad(s \in[0,1]) .
$$

Wegen (17) haben wir

$$
f(0)=h^{*} Q x_{1}=x \varrho h^{*} u>0
$$

und wegen (20)

$$
f(1)=h^{*}\left[Q x_{0}+q \varphi\left(t_{0}, r^{*} x_{0}\right)\right]<0 .
$$

Wegen der Stetigkeit von $f$ existiert laut Zwischenwertsatz ein $\bar{s} \in(0,1)$ mit $f(\bar{s})=0$. Aus letzterem ergibt sich jedoch wegen (8) und (23) die Gleichung

$$
Q y(\bar{s})+q \tilde{\varphi}\left(r^{*} y(\bar{s})\right)=0 \text {. }
$$

Offensichtlich widersprechen sich die Beziehungen (24) und (25). Damit ist die Einschließung (15) bewiesen. Die übrigen Aussagen des Lemmas lassen sich analog zeigen 
Die nächste Behauptung findet man für verschiedene spezielle Funktionen $V(x)$ implizit in einer Reihe von Arbeiten [1, 5-7].

Lemma 3. Es mögen für das System

$$
x_{t+1}=\mathfrak{f}\left(x_{t}, t\right) \quad(t=0,1, \ldots)
$$

mit der v-dimensionalen Vektorfunktion $\mathfrak{f}(x, t)\left(x \in \mathbf{R}^{*}, t=0,1, \ldots\right)$ eine Funktion $V(x)\left(x \in \mathbf{R}^{\prime}\right)$, eine ganze Zahl $t_{0} \geqq 0$, eine Zahl $\lambda$ mit $0<\lambda \leqq 1$ und eine Zahl $\delta>0$ existieren, für die auf den Lösungen $x_{t}$ des Systems (26) die Ungleichung

$$
\frac{1}{\lambda^{2}} V\left(x_{t+1}\right)-V\left(x_{t}\right) \leqq-\delta\left|x_{t}\right|^{2} \quad\left(t \geqq t_{0}\right)
$$

gilt. Dann gelangt jede nicht gegen Null konvergierende Lösung $x_{\ell}$ von System (26) in die Menge $\Gamma=\{x \mid V(x)<0\}$ und verbleibt dort.

Beweis. Nach Umformung von (27) erhält man

$$
V\left(x_{t+1}\right)-V\left(x_{t}\right)+\left(1-\lambda^{2}\right) V\left(x_{t}\right) \leqq-\lambda^{2} \delta\left|x_{t}\right|^{2} \quad\left(t \geqq t_{0}\right) .
$$

Es sei $x_{t}$ eine beliebige Lösung von (26), die nicht gegen Null konvergiert und für die folglich

$$
\sum_{t=t_{\bullet}}^{\infty}\left|x_{t}\right|^{2}=+\infty
$$

gilt. Es werde angenommen, daB diese Lösung nicht in die Menge $\Gamma$ gelangt, d. h., es sei

$$
\nabla\left(x_{t}\right) \geqq 0 \quad\left(t \geqq t_{0}\right) .
$$

Dann kann wegen (30) die Ungleichung (28) umgeformt werden zu

$$
V\left(x_{t+1}\right)-V\left(x_{t}\right) \leqq-\lambda^{2} \delta\left|x_{t}\right|^{2} \quad\left(t \geqq t_{0}\right) .
$$

Wird nun die letzte Beziehung von $t=t_{0}$ bis $t=T$ summiert, so erhält man

$$
V\left(x_{T+1}\right)-V\left(x_{t_{0}}\right) \leqq-\lambda^{2} \delta \sum_{t=t_{0}}^{T}\left|x_{\ell}\right|^{2} .
$$

Nun ergibt sich aber aus (31) unter Berücksichtigung von (29) sofort $V\left(x_{t}\right) \rightarrow-\infty$ $(t \rightarrow+\infty)$, was der Voraussetzung (30) widerspricht. Folglich gelangt jede Lösung von (26), die nicht gegen Null konvergiert, in $\Gamma$ und verbleibt wegen (27) dort

Lemma 4. Es sei für die $\nu \times \nu$-Matrix $H=H^{*}$ und den $\nu$-Vektor h die Beziehung (8) erfuill. Dann ist für beliebige Zahlen $\alpha_{1}$ und $\alpha_{2}$ mit $\alpha_{1} \leqq \alpha_{2}$ die Menge

$$
M=\left\{x \mid x^{*} H x \leqq 0\right\} \cap\left\{x \mid \alpha_{1} \leqq h^{*} x \leqq \alpha_{2}\right\}
$$

beschränkt.

Beweis. Aus [13] (Hilfssatz 1) folgt, daß unter den Bedingungen des Lemmas die Menge $M$ konvex ist. Es werde angenommen, da $B M$ unbeschränkt ist. Wegen der Konvexität von $M$ bedeutet diese Annahme, daß ein Strahl existiert, der vollständig in $M$ liegt, d. h., daß zwei Vektoren $a, d \in \mathbf{R}^{\prime}(d \neq 0)$ existieren, für die gilt: $a+s d \in M(s \geqq 0)$. Aus letzterem folgt aber unter Berücksichtigung der Konstruktion der Menge $M$, da $B h^{*} d=0$ und daß wegen (8) $d=0$ ist. Der erhaltene Widerspruch beweist das Lemma

Bemerkung. Im Prinzip ist die Aussage von Lemma 4 implizit in einer Reihe von Arbeiten enthalten [4,8]. 
Beweis von Satz 1. Da das Paar $\left(\frac{1}{\lambda} A, b\right)$ vollständig steuerbar ist, die Matrix $\frac{1}{\lambda} A$ keinen Eigenwert auf dem Einheitskreis hat und die Frequenzungleichungen in Satz 1 als erfüllt vorausgesetzt werden, existieren nach dem Frequenzsatz [16] eine $\nu \times \nu$-Matrix $H=H^{*}$ und eine Zahl $\delta>0$, so daß gilt :

$\frac{1}{\lambda^{2}}(A x+b \xi)^{*} H(A x+b \xi)-x^{*} H x+\mathcal{G}\left(c^{*} x, \xi\right) \leqq-\delta|x|^{2}\left(x \in \mathbf{R}^{*}, \xi \in \mathbf{R}^{1}\right)$.

Dabei ist die Funktion $\left(\mathcal{H}(\sigma, \xi)\left(\sigma \in \mathbf{R}^{1}, \xi \in \mathbf{R}^{1}\right)\right.$ durch (14) erklärt. Wird in (32) $\xi=0$ gesetzt und beachtet, $\operatorname{da} B\left(\mathcal{H}^{*} x, 0\right) \geqq 0\left(x \in \mathbf{R}^{*}\right)$ ist, so erhält man die Ungleichung

$$
\frac{1}{\lambda^{2}} x^{*} A^{*} H A x-x^{*} H x \leqq-\delta|x|^{2} \quad\left(x \in \mathbf{R}^{\prime}\right)
$$

Da die Matrix $\frac{1}{\lambda} A$ einen Eigenwert außerhalb des Einheitskreises und $v-1$ Eigenwerte innerhalb des offenen Einheitskreises besitzt, so folgt wegen (33) und [15], $\mathrm{da} B$ die Matrix $\boldsymbol{H}$ einen positiven und $\boldsymbol{\nu}-1$ negative Eigenwerte besitzt. Weiter existiert nach [4] ein Vektor $h$, so da $B$ für das Paar $H, h(8)$ erfüllt ist. Wir definieren die Funktionen

$$
V_{j}(x)=(x-j r)^{*} H(x-j r) \quad\left(x \in \mathbf{R}^{\prime}\right)
$$

für beliebige ganze Zahlen $j$ ( $r$ ist der Vektor aus (5)). Weiterhin werden für diese $j$ die folgenden Mengen eingefüht:

und

$$
\Gamma_{j}=\left\{x \mid V_{j}(x)<0\right\}, \quad \Gamma_{j}^{+}=\Gamma_{j} \cap\left\{x \mid h^{*}(x-j r) \geqq 0\right\}
$$

$$
\Gamma_{j}^{-}=\Gamma_{j} \cap\left\{x \mid h^{*}(x-j r) \leqq 0\right\}
$$

Wird in der Ungleichung (33) $x=r$ gesetzt, so erhält man $\left(\frac{1}{\lambda^{2}}-1\right) r^{*} H r \leqq-\delta|r|^{2}$
und folglich ist

$$
r * H r<0 .
$$

Wir betrachten nun eine beliebige Lösung $x_{\ell}\left(t_{0}, x_{0}\right)$ des Systems (1). Für deren Verhalten gibt es zwei prinzipielle Möglichkeiten:

1. Es existiert eine ganze Zahl $j$, so daß $x_{t}\left(t_{0}, x_{0}\right) \rightarrow j r(t \rightarrow+\infty)$ gilt. Eine solche Lösung ist offensichtlich beschränkt.

2. Die Lösung strebt zu keinem $j r$. Wir beweisen nun, daß eine solche Lösung von einem bestimmten Zeitpunkt an in allen Mengen $\Gamma_{j}$ liegt. Mit diesem Ziel betrachten wir den Ausdruck

$$
V_{j}\left(x_{0}\right)=\left(x_{0}-j r\right)^{*} H\left(x_{0}-j r\right)=x_{0}^{*} H x_{0}-2 j r^{*} H x_{0}+j^{2} r^{*} H r
$$

Aus (34) folgt dann, daß eine ganze $Z a h l j_{0}>0$ existiert, so daß die Ungleichung $V_{j}\left(x_{0}\right)<0$ für alle $j$ mit $|j| \geqq j_{0}$ erfüllt ist. Nun betrachten wir eine beliebige ganze Zahl $j$ mit $|j|<j_{0}$. Da $x_{t}\left(t_{0}, x_{0}\right) \rightarrow j r(t \rightarrow \infty)$ gilt, erhält man (unter Beruicksichtigung der Periodizität von $\varphi$ ):

$$
x_{t}\left(t_{0}, x_{0}\right)-j r=x_{t}\left(t_{0}, x_{0}-j r\right) \nrightarrow 0 \quad(t \rightarrow+\infty) .
$$


Aus (32) folgt die Ungleichung

$\frac{1}{\lambda^{2}} V_{0}\left(x_{\ell+1}\left(t_{0}, x_{0}-j r\right)\right)-V_{0}\left(x_{t}\left(t_{0}, x_{0}-j r\right)\right) \leqq-\delta\left|x_{t}\left(t_{0}, x_{0}-j r\right)\right|^{2} \quad\left(t \geqq t_{0}\right)$.

Wegen Lemma 3 ergibt sich aus der letzten Ungleichung, da $B$ ein Zeitpunkt $\iota_{1}$ existiert, so daß $x_{t}\left(t_{0}, x_{0}-j r\right) \in \Gamma_{0}$, d. h. $x_{t}\left(t_{0}, x_{0}\right) \in \Gamma_{j}\left(t \geqq t_{1}\right)$ gilt. Da nur für eine endliche Anzahl von ganzen $j$ die Bedingung $|j|<j_{0}$ erfüllt ist, läßt sich ein solches $t_{1}$ auswählen, das einheitlich für alle diese $j$ ist. Demzufolge kann man annehmen, ohne dabei die Allgemeinheit zu verletzen, $\mathrm{da} \beta x_{t}\left(t_{0}, x_{0}\right)$ eine Jösung ist, für die $x_{0} \in \Gamma_{\text {; }}$ für alle ganzen Zahlen $j$ gilt. Nun läßt sich ein $j_{1}$ angeben, für das $j_{1} h^{*} r$ $\leqq h^{*} x_{0} \leqq\left(j_{1}+1\right) h^{*} r$ ist. (Es ist $h^{*} r \neq 0$ wegen (8) und (34).) Also gilt auch

$$
x_{0} \in F_{j_{1}}:=\Gamma_{j_{1}} \cap \Gamma_{j_{1}+1} \cap\left\{x \mid j_{1} h^{*} r \leqq h^{*} x \leqq\left(j_{1}+1\right) h^{*} r\right\}=\Gamma_{j_{2}}^{+} \cap \Gamma_{j_{1}+1}^{-} .
$$

Mit Lemma 4 folgt aus (8) die Beschränktheit der Menge $F_{j_{i}}$. Als nächstes wird die Invarianz von $F_{j_{1}}$ bezüglich der Lösungen des Systems (1) bewiesen, d. h., wir zeigen :

$$
x_{t}\left(t_{0}, x_{0}\right) \in F_{j_{1}} \quad\left(t \geqq t_{0}\right) .
$$

Wegen $x_{0} \in \Gamma_{j_{1}}^{+}$gilt $x_{0}-j_{1} r \in \Gamma_{0}^{+}$. Aus Lemma 2 folgt die Inklusion $x_{t}\left(t_{0}, x_{0}-j_{2} r\right) \in \Gamma_{0}^{+}$ $\left(t \geqq t_{0}\right)$. Demzufolge ist

$$
x_{t}\left(t_{0}, x_{0}\right) \in \Gamma_{j_{1}}^{+} \quad\left(t \geqq t_{0}\right) .
$$

Andererseits ist $x_{0} \in \Gamma_{j+1}^{-}$. Folglich gilt $x_{0}-\left(j_{1}+1\right) r \in \Gamma_{0}^{-}$. Wegen Lemma 2 erhält man $x_{t}\left(t_{0}, x_{0}+\left(j_{1}-1\right) r\right) \in \Gamma_{0}^{-}$und, demzufolge,

$$
x_{i}\left(t_{0}, x_{0}\right) \in \Gamma_{\overline{j_{i}+1}}^{-} \quad\left(t \geqq t_{0}\right) \text {. }
$$

Aus (37) und (38) folgt sofort (36). Der Satz 1 ist damit vollständig bèwiesen

Definition [7]. Das System (1) heißt $\Delta$-stabil im ganzen, wenn für eine beliebige Lösung $x_{t}$ des Systems (1) eine Zahl $t_{0}$ existiert, so daß gilt :

$$
\left|c^{*} x_{t_{1}}-c^{*} x_{t_{\mathrm{g}}}\right| \leqq \Delta \quad\left(t_{1} \geqq t_{0}, t_{2} \geqq t_{0}\right) .
$$

Satz 2. Es seien alle Bedingungen von Satz 1 erfüll; dabei sei entweder $\mu_{1}=-\infty$ oder $\mu_{2}=+\infty$. Dann ist System (1) $\Delta$-stabil im ganzen.

Beweis. Es läßt sich der gesamte Beweis von Satz 1 wiederholen. Außerdem folgt aus (32) mit Lemma 1 die Beziehung

$$
\left\{x \mid x^{*} H x \leqq 0\right\} \cap\left\{x \mid c^{*} x=0\right\}=\{0\} .
$$

Deshalb kann anstelle von $h$ im Beweis von Satz 1 der Vektor $c$ genommen werden. Aus dem Beweis von Satz 1 folgt, daß die Mengen

$$
F_{i}:=\Gamma_{j} \cap \Gamma_{j+1} \cap\left\{x \mid j \Delta \leqq h^{*} x \leqq(j+1) \Delta\right\}
$$

invariant für die Lösungen von System (1) sind und jede Lösung, die nicht zu einem der $j r$ strebt, in eine der Mengen $F_{j}$ gelangt. Hieraus erhält man sofort die Behauptung ron Satz 2

Bemerkung. Im Beweis der Sätze 1 und 2 wird die Methode der Konstruktion eines Netzes von invarianten quadratischen Kegeln benutzt, wie sie in den Arbeiten $[9-11,5,7]$ verwendet wurde. Die Beschränktheit der Lösungen diskreter Phasensysteme wird in $[1,5]$ unter der Voraussetzung gezeigt, da $\varphi(t, \sigma) \equiv \varphi(\sigma)$ eine stetige Funktion ist. Im Unterschied dazu ist im Satz $1 \varphi(t, \sigma)$ eine beliebige skalare Funktion, die einer gewissen Sektorbedingung genügt. 
In der Arbeit [7] werden Frequenzbedingungen für $\Delta$-Stabilität im ganzen von diskreten Phasensystemen angegeben, wobei Lemma 1 aus [13] verwendet wird. Im Unterschied zu Satz 2 der vorliegenden Arbeit wird in [7] zusätzlich vorausgesetzt, $\operatorname{daB} \varphi(t, \sigma) \equiv \varphi(\sigma)$ ist und $\varphi(\sigma)$ eine stückweise stetige Funktion darstellt, die eine endliche Anzahl von Unstetigkeitsstellen erster Art auf dem Intervall $[0, \Delta]$ hat und diese folgender Bedingung genügt: Wenn $\sigma_{1} \in[0, \Delta), \sigma_{2} \in[0, \Delta]$ mit $\sigma_{1}<\sigma_{2}$ beliebige Unstetigkeitsstellen von $\varphi(\sigma)$ sind, wobei $\varphi(\sigma)$ stetig für $\sigma \in\left(\sigma_{1}, \sigma_{2}\right)$ ist, so existiert ein Punkt $\sigma^{\prime} \in\left(\sigma_{1}, \sigma_{2}\right)$, so da $0 \leqq \sigma^{\prime} \leqq c^{*} b \varphi\left(\sigma^{\prime}\right) \leqq \Delta$ gilt. Unstetige Nichtlinearitäten spielen eine große Rolle z. B. bei Ziffernsystemen der Phasensynchronisation [14].

Als nächstes wird noch ein Satz über die Beschränktheit der Lösungen von Phasensystemen formuliert, der keinen Frequenzcharakter trägt. Der Beweis ist, wie auch oben, mit der Konstruktion eines.Kegelnetzes im $\mathbf{R}^{\prime}$ verbunden. Im folgenden Satz werden die Ungleichungen $B>0(B \geqq 0)$, wobei $B$ ein Vektor oder eine Matrix ist, so verstanden, da $\beta$ alle Komponenten von $B$ positiv (nichtnegativ) sind.

Satz 3. Es sei die Ungleichung (2) mit $\mu_{2}=+\infty$ erfïllt. Ess sei außerdem $A>0$, $b \geqq 0, c \geqq 0$ und $A+\mu_{1} b c^{*} \geqq 0$. Dann ist jede Lösung des Systems (1) beschränkt auf $[0,+\infty)$.

Beweis. Da die Matrix $A$ den Eigenwert 1 besitzt und $A>0$ ist, kann man annehmen, daß (5) mit einem Vektor $r>0$ erfüllt ist. Wir definieren für ein beliebiges ganzes $j$ die Mengen

$$
K_{j}^{+}=\{x \mid x \geqq j r\} \quad \text { und } \quad K_{j}^{-}=\{x \mid x \leqq j r\} .
$$

Es ist offensichtlich, daß für beliebiges $j$ diese Mengen konvex sind; folglich ist auch die Menge $\Gamma_{j_{1}, j_{1}}=K_{j_{1}}^{+} \cap K_{\widetilde{j_{2}}}$ mit $j_{1}<j_{2}$ konvex. Die Menge $\Gamma_{j_{1}, j_{1}}$, ist beschränkt: Wäre dies nicht so, so existierte wegen ihrer Konvexität ein Strahl, der vollkonmen dieser Menge angehört, d. h., es müßten solche Vektoren $a, q \in \mathbf{R}^{\prime}$ mit $q \neq 0$ existieren, so daß $a+s q \in \Gamma_{j_{1}, j,}(s \geqq 0)$ gilt. Aus der letzten Einschließung erhält man jedoch die Ungleichungen $j_{1} r \leqq a+s q \leqq j_{2} r(s \geqq 0)$. Hieraus folgt sofort $q=0$.

Sei $x_{0}$ ein beliebiger Punkt. Da $r>0$ ist, existieren solche ganze Zahlen $j_{1}$ und $j_{2}$, daß $j_{1} r \leqq x_{0} \leqq j_{2} r$ gilt. Wir beweisen nun, da $x_{t}\left(t_{0}, x_{0}\right) \in \Gamma_{j_{1}, j_{s}}\left(t \geqq t_{0}\right)$ ist. Letzteres ist bewiesen, wenn gezeigt wird, daß gilt:

$$
x_{t}\left(t_{0}, x_{0}\right) \in K_{\dot{j}_{1}}^{+} \quad \text { und } \quad x_{t}\left(t_{0}, x_{0}\right) \in K_{j_{2}}^{-} \quad\left(t \geqq t_{0}\right) .
$$

Ihrerseits ist die Beziehung (39) gezeigt, wenn die Einschließungen $x_{\ell}\left(t_{0}, x_{0}\right)-j_{1} r \in K_{0}{ }^{+}$ und $x_{t}\left(t_{0}, x_{0}\right)-j_{2} r \in K_{0}^{-}, \mathrm{d}$. h.

$$
x_{t}\left(t_{0}, x_{0}-j_{1} r\right) \in K_{0}^{+} \quad \text { und } \quad x_{t}\left(t_{0}, x_{0}-j_{2} r\right) \in K_{0}^{-} \quad\left(t \geqq t_{0}\right)
$$

gelten. Die Beziehung (40) ist dazu äquivalent, daß für ein beliebiges $y_{0} \in K_{0}{ }^{+}$und ein beliebiges ganzes $t_{1} \geqq 0$ die Beziehung $A y_{0}+b \varphi\left(t_{1}, c^{*} y_{0}\right) \in K_{0}{ }^{+}$gilt und für ein beliebiges $y_{0} \in K_{0}^{-}$und ein beliebiges ganzes $t_{1} \geqq 0$ die Beziehung $A y_{0}+b \varphi\left(t_{1}, c^{*} y_{0}\right)$ $\in K_{0}{ }^{-}$gilt. Es sei zunächst $y_{0} \in K_{0}{ }^{+}$und $t_{1} \geqq 0$. Wir benutzen die folgende Gleichung:

$$
y_{1}={ }^{\circ} A y_{0}+b \varphi\left(t_{1}, c^{*} y_{0}\right)=\left(A+\mu_{1} b c^{*}\right) y_{0}+b\left[\varphi\left(t_{1}, c^{*} y_{0}\right)-\mu_{1} c^{*} y_{0}\right]
$$

Aus der Bedingung (2) folgt die Ungleichung $\varphi\left(t_{1}, c^{*} y_{0}\right)-\mu_{1} c^{*} y_{0} \geqq 0$. Also ist $y_{1} \geqq 0$. Es sei nun $y_{0} \in K_{0}-$ und $t_{1} \geqq 0$. Wiederum benutzen wir die Gleichung (41). Wegen (2) und $c^{*} y_{0} \leqq 0$ ist $\varphi\left(t_{1}, c^{*} y_{0}\right)-\mu_{1} c^{*} y_{0} \leqq 0$ und damit $y_{1} \leqq 0$. Demzufolge ist die Invarianz der Menge $\Gamma_{j_{1}}, j_{2}$ bezüglich der Lösungen von System (1) gezeigt. Aus ihr erhält man die Beschränk theit dieser Lösungen, d. h. die Aussage des Satzes 
Beispiel. Wir betrachten ein autonomes Ziffernphasenkopplungssystem erster Ordnung, in dem in der Steuerkette der Ziffernfilter fehlt [14]:

$$
\Theta[n+1]-\Theta[n]=\Omega_{H} T_{P}-\Omega_{Y} T_{P} F(\Theta[n]) \quad(n=0,1, \ldots) .
$$

Hierbei sind $T_{P}$ die Folgezeit des Signals des Phasenfehlers, $\Omega_{Y}$ der Haltebereich, $\Omega_{H}$ die Anfangsdifferenz der Frequenzen des abzustimmenden Generators und des Eichgenerators bei Nichtvorhandensein von Phasenmodulation des Eichgenerators. Weiterhin ist $F(\Theta)$ eine normierte $2 \pi$-periodische Charakteristik des Ziffern-Phasendetektors. Es wird vorausgesetzt, daß für $F(\Theta)$ gilt:

$$
F(\Theta)= \begin{cases}+1 & \text { für } 0 \leqq \Theta<\pi \\ -1 & \text { für },-\pi \leqq \Theta<0 .\end{cases}
$$

Wird (43) in (42) eingesetzt und die Größe $\gamma_{H}=\Omega_{H} / \Omega_{Y}$ eingeführt, so erhält man die Gleichung

mit

$$
\Theta[n+1]=\Theta[n]+F_{1}(\Theta[n]) \quad(n=0,1, \ldots)
$$

$$
F_{1}(\Theta)=\left\{\begin{array}{lll}
\Omega_{Y} T_{P}\left(\gamma_{H}-1\right) & \text { für } \quad 0 \leqq \Theta<\pi \\
\Omega_{Y} T_{P}\left(\gamma_{H}+1\right) & \text { für } \quad-\pi \leqq \Theta<0 .
\end{array}\right.
$$

Wird schließlich noch $\Theta[n]=\sigma[n]+\pi$ gesetzt, ergibt sich das System

$$
\sigma[n+1]=\sigma[n]+F_{2}(\sigma[n]) \quad(n=0,1, \ldots),
$$

wobei $F_{2}(\sigma) \equiv F_{1}(\sigma+\pi)$ ist. Wir untersuchen System (44) zunächst mit Satz 2. Die Ubertragungsfunktion des linearen Teils hat die Form $\chi(p)=\frac{1}{1-p}$. Es sei
$\left|\gamma_{H}\right|<1$. Dann ist leicht zu sehen, daß durch die Festlegung

$$
\mu_{1}=\min \left\{\frac{\Omega_{Y} T_{P}}{\pi}\left(\gamma_{H}-1\right), \quad \frac{-\Omega_{Y} T_{P}}{\pi}\left(\gamma_{H}+1\right)\right\}
$$

die Ungleichung

$$
F_{2}(\sigma) \sigma \geqq \mu_{1} \sigma^{2} \quad\left(\sigma \in \mathbf{R}^{1}\right)
$$

gilt. Für beliebige $\alpha \in[-\pi, \pi]$ und $\lambda \in(0,1)$ erhält man

und

$$
\operatorname{Re} \chi\left(\lambda \mathrm{e}^{i \alpha}\right)=\frac{1-\lambda \cos \alpha}{(1-\lambda \cos \alpha)^{2}+\lambda^{2} \sin ^{2} \alpha}
$$

$$
\left|\chi\left(\lambda \mathrm{e}^{i \alpha}\right)\right|=\frac{1}{(1-\lambda \cos \alpha)^{2}+\lambda^{2} \sin ^{2} \alpha} .
$$

Demzufolge ist die Frequenzbedingung aus Satz 2 erfüllt, wenn $1-\lambda \cos \alpha+\mu_{1}>0$ $(\alpha \in[-\pi, \pi)]$ gilt. Da wir hier $\lambda \in(0,1)$ beliebig wählen können, ist die letzte Ungleichung erfüllt, wenn $1+\mu_{1}>0$ ist, d. h., wenn gilt

$$
\max \left\{\frac{-\Omega_{Y} T_{P}}{\pi}\left(\gamma_{H}-1\right), \quad \frac{\Omega_{Y} T_{P}}{\pi}\left(\gamma_{H}+1\right)\right\}<1 .
$$

Unter diesen Voraussetzungen ist dann laut Satz 2 das System (44) (und folglich auch das System (42)) $2 \pi$-stabil im ganzen. Wir wenden nun auf System (44) den Satz 3 an. Mit den Bezeichnungen dieses Satzes gilt:

$$
A=1>0, \quad c=b=1>0, \quad A+\mu_{1} b c^{*}=1+\mu_{1} .
$$


Wird nun gefordert, daß

$$
\max \left\{\frac{-\Omega_{Y} T_{P}}{\pi}\left(\gamma_{H}-1\right), \quad \frac{\Omega_{Y} T_{P}}{\pi}\left(\gamma_{H}+1\right)\right\} \leqq 1
$$

gilt, so sind alle Bedingungen von Satz 3 erfüllt, und demzufolge hat System (44) (und folglich auch System (42)) nur beschränkte Lösungen.

Man sieht also, daß die Sätze 2 und 3 bezüglich der Beschränktheit der Lösungen von System (42) faktisch den gleichen Parameterbereich beschreiben. Allerdings garantiert Satz 2 darüber hinaus die $2 \pi$-Stabilität im ganzen.

\section{LITERATUR}

[1] Abramovič, S., Yu. Koryakin, G. Leonov, und V. Reitsiann: Frequenzbedingungen für Schwingungen in diskreten Systemen. II. Schwingungen in diskreten Phasensystemen. Wiss: Z. der TU Dresden 26, H. 1 (1977), 115-122.

[2] Андронов, А. А., А. А. Витт, и С. Э. ХАЙкин: Теория колебаний. Москва 1959.

[3] Белых, В. Н., и В. П. МАксАков: Динамика ПростеЙеЙ дискретной системы Фазовой синхронизации. Радиотехника и әлектроника 21 (1976), 2155-2163.

[4] Гелиг, А. Х., Г. А. ЛЕонов, и В. А. Якувович: Устоичивость нелинейных систем с неединственным состоянием равновесия. Москва 1978.

[5] Корякин, Ю. А.: Некоторые вопросы динамики дискретных фазовых систем. Диссертация, Ленинград 1977.

[6] Корякин, Ю. А., и Г. А. ЛЕонов: Определение полосы аахвата в системах импульсно-фазовой автоподстройи частоты. Раднотехника. 32, № 6 (1977), 65-72.

[7] Корякин, Ю. А., Г. А. ЛЕонов, и А. Р. Лисс: Частотиый критерий устойчивости дискретных систем автоматического управления фазой колебанй генератора. Автомат. и телемех. 12 (1978), 64-69.

[8] Koryaktn, Yo. A., G. A. Leonov und V. Rertmann: Konvergenz im Mittel von Phasensystemen. ZAMOI 58 (1978), 435-441.

[9] ЛЕонов, Г. А.: Об устойчивости фазовых систем. Сиб. мат. журн. 15 (1974), 49-60.

[10] Лвонов, Г. А.: Об ограниченности траекторий фазовых систем. Сиб. мат. журн. $15(1974), 687-692$.

[11] ЛЕонов, Г. А.: Устойивость и колебания фазовых систем. Сиб. мат. журн. 16 (1975), $1031-1052$.

[12] РАйтан, Ф.: Частотные условия колебательности и неустойчивости дискретных систем автоматіческого управления. Диссертадия, Ленинград 1980.

[13] Reitma NN, V.: Uber Instabilität im ganzen von nichtlinearen diskreten Systemen. ZAMOM 59 (1979), 652-655.

[14] ШІ Ахгильдян, В. В. (ред.): Системы фазовои автоподстройки частоты с элементами дискретивадии. Москва 1979.

[15] Шепелявыћ, А. И.: Абсолютная неустоичивость нелинейных амплитудно-импульсных систем управления. Частотные критерии. Автомат. и телемех. 6 (1972), 49-56.

[16] Якувович, В. А.: Частотная теорема в теории управления. Сиб. мат. журн. 14 (1973), $384-420$.

Manuskripteingang: 8. 10. 1980

\section{VERFASSER:}

VOLKER REITMANN

Sektion Mathematik/WB Numer. Math. der Technischen Universität

DDR - 8027 Dresden, Mommsenstr. 13 